\title{
CIUDADANÍA Y ÉTICA EMPRESARIAL: ANÁLISIS CRÍTICO Y CONTRIBUCIONES PARA UN PROYECTO DE PARTICIPACIÓN ECONÓMICA*
}

\author{
Pablo Gres C.** \\ Universidad de Chile, Chile \\ pablogres@ug.uchile.cl
}

RESUMEN: Este trabajo tiene como objetivo principal criticar la propuesta de ética de la empresa como práctica de la ciudadanía económica. Me centraré particularmente en el proyecto de la Dra. Adela Cortina.

Para dar un orden coherente a la investigación, la he dividido en tres grandes partes. El primer tópico se titula Ética y economía en la historia de la filosofía, donde se explica la posible relación entre estas dos disciplinas, tomando como punto de partida el inicio del capitalismo, el objetivo es poder poner en un contexto adecuado el siguiente apartado. En segundo lugar se explicará el proyecto de la ética empresarial propuesto por Adela Cortina, siempre en el marco de su propuesta ciudadana. Finalmente se realizara una crítica para poder esbozar un proyecto de participación -económica- radical.

Palabras clave: Ciudadanía, mercado, participación, ética.

\section{CITIZENSHIP AND BUSINESS ETHICS: CRITICAL ANALYSIS AND CONTRIBUTIONS TO AN ECONOMIC PARTICIPATION PROYECT}

\begin{abstract}
This article has as its main purpose to criticize the proposed business ethics as practice of economical citizenship. I will focus particularly on the project of Dr. Adela Cortina. To give a logical order, I have divided it into three parts. The first topic is titled Ethics and economics in the philosophy's history, where I explain the possible relationship between these two disciplines, taking the beginning of capitalism
\end{abstract}

Trabajo recibido el 19 de enero y aprobado el 17 de marzo de 2012.

** Estudiante de Derecho, Universidad de Chile. Alumno ayudante de las cátedras de Filosofía (de la) Moral y Derecho Constitucional. 
as its starting point. The main objective is to put in proper context our next section. The second topic, explains the business ethics project proposed by Adela Cortina, always in the context of her citizens' proposal. Finally, I will make a critic in order to outline a proposal of a radical economic participation.

Keywords: citizenship, market, participation, ethics.

\section{INTRODUCCIÓN}

Desde que el capitalismo existe como tal, grandes pensadores se han preocupado de construir una relación convincente entre ética, economía y empresa, tal es el caso de Adam Smith, John Stuart Mill o Max Weber, entre otros.

En los años 70 del siglo XX, debido a la pérdida de la confianza en las empresas por parte de la ciudadanía, surge, junto a todo un gru po de éticas aplicadas, la ética de la empresa.

Este trabajo busca presentar el proyecto ético-filosófico de la ética de la empresa, principalmente el propuesto por la profesora Adela Cortina, debido a que a mi juicio es el más completo. En primer lugar explicaré en qué sentido ésta se enmarca dentro de la ética aplicada y ética ciudadana, además de aclarar a que refieren con tales conceptos. Me detendré un momento, para presentar las teorías filosóficas, que me parece, han sido más influyentes y más importantes respecto de la relación ética, economía y empresa. Lo que busco constatar en este apartado es que ética y economía no son dos disciplinas excluyentes. Posteriormente, me referiré a cómo la ética empresarial se fundamenta, principalmente en la ética discursiva, y cuál es su proyecto de acción, para relacionar esto con lo que ya se ha expuesto sobre la ética aplicada y cívica. Finalmente, al ser éste un estudio crítico, me detendré un momento para referirnos a los puntos en que estamos de acuerdo con el proyecto de la Dra. Cortina, pero elaboraremos una crítica desde la teoría del reconocimiento, basada en el pensamiento hegeliano con el objetivo de contribuir y apuntar a un proyecto de participación radical.

\section{2. ÉTICA Y ECONOMÍA EN LA HISTORIA DE LA FILOSOFÍA}

Desde que el capitalismo comenzó a funcionar industrialmente, pensadores y teóricos liberales se ocuparon de forjar una relación, al menos teórica, entre ética y economía ${ }^{1}$. El ejemplo más bullado corresponde a la edad industrial del capitalismo, nos referimos al esfuerzo de Adam

\footnotetext{
Soy consciente de que el concepto que usan los teóricos liberales, al hacer la relación entre economía y ética, no es precisamente el de empresa, sino que el de enterprise, que traducido al español es emprendedor. Dicho concepto ha sido objeto de un cierto revival, por lo menos en nuestro país. Nos parece que la razón de aquello es simple: el concepto de emprendedor pareciera no tiene una carga política e ideológica tan fuerte como el de empresa (al menos en un primer momento), en términos de que todos aquellos que participan del juego del mercado pueden ser considerados emprendedores.
} 
Smith por crear la relación entre economía y ética. En la Teoría de los sentimientos morales y en La riqueza de las naciones, ambas obras centrales en el pensamiento del escocés y de la teoría liberal clásica, el autor busca crear una relación desde la teoría de la utilidad, dar un respaldo ético a una teoría económico-política, un respaldo fundado en la simpatía, el amor propio y el afán de lucro, entre otros sentimientos, que en definitiva son el motor del intercambio y permiten entender el por qué de la actividad económica.

En la misma línea de argumentación podemos encontrar a grandes teóricos del liberalismo como David Hume, John Locke o Jeremy Bentham. Un caso bastante particular que a nuestro juicio merece detención y análisis es el de John Stuart Mill.

John Stuart Mill fue educado en el seno de la teoría liberal y positivista. En su obra, se ocupa de negar cualquier fundamento de la ética que no fuese la humanidad misma. Nos parece que las obras centrales que permiten crear la relación entre economía y ética, son los Principios de Economía-Política: con alguna de sus aplicaciones a la filosofia social, El Utilitarismo y Sobre la Libertad. El punto que brevemente queremos desarrollar acá es simple: si bien Mill nunca abandonó el argumento liberal de corte y fundamento utilitarista, se preocupó de reformularlo. Lo que determina la utilidad en Mill es el principio de máxima felicidad, que apunta a aumentar la felicidad y disminuir o eliminar el dolor, pero superpone los placeres intelectuales por sobre los corporales, a demás de dar más importancia a la utilidad que puede producir un trabajo más que a la riqueza entendida como posibilidad de acumulación. En tal sentido Mill es el primer punto de inflexión dentro de los teóricos liberales.

Otra teoría que busca mostrar la relación entre ética y empresa es la que Max Weber desarrolla en La ética protestante y el espiritu del capitalismo. En resumidas cuentas, sin ánimo de banalizarlo, el argumento de Max Weber puede explicarse del siguiente modo: "intentó mostrar cómo el espíritu del capitalismo procedió a su encarnadura económica, cómo un tipo de ética la ética protestante en este caso - conformaba ese espíritu que alentó el cuerpo del capitalismo al nacer" 2 . Desde la ética protestante, en especifico desde el calvinismo pueden ser "justificados éticamente, no sólo el trabajo, sino también la acumulación consistente y legal de riqueza"3.

Me parece que la formulación de Weber es merecedora de varios reparos. En primer lugar, nos gustaría señalas que deja de tener sentido desde el momento en que culturas o países tan extrańos al protestantismo como Japón, han interiorizado el capitalismo como parte de ellos. Por otro lado Martín Hopenhayn señala que la ética protestante tiene importancia en el desarrollo capitalista pero afirma que "[n]o sólo el ascetismo puritano estimuló la acumulación de capital necesaria para hacer posible la transición del capitalismo comercial e industrial; el propio capitalismo comercial creó condiciones para tal acumulación. La afluencia de metales

2 Cortina, Adela. Construir confianza. Ética de la empresa en la sociedad de la información y las comunicaciones. Madrid, España: Trotta, 2004, p. 24.

$3 \quad$ Ibid., p. 24-25. 
preciosos americanos a Europa aumentó el circulante, generando un alza en los precios que contribuyó al desarrollo del capitalismo"”. Esa creación de condiciones que señala Hopenhayn es la idea de Galeano en Las venas abiertas de América latina, reformulada en términos académicos y formales.

En este punto del análisis se hace necesario recordar el proyecto de Kant, debido a que siendo un liberal rompe radicalmente con el fundamento utilitarista e individualista anglosajón de la ética, y aunque su obra no versa sobre economía es el fundamento de formulaciones actuales que relacionan economía y ética. Entre otras cosas Kant propone la universalización de las normas, una ética deontológica y la no instrumentalización del otro.

Desde mediados del siglo XIX, se produce un fenómeno bastante interesante. Las humanidades comenzaron a convertirse en Ciencias Sociales. En tal sentido la Economía se convierte en Economía Científica (al menos desde J. S. Mill en adelante), lo demás es Economía Política. La Economía en términos científicos comienza a ocuparse de los precios (ya no del valor ${ }^{5}$ ) de los productos, de la circulación (en vez de la producción) y de los cálculos económicos concretos, de las variaciones de precio, lo que entrega a esta disciplina cierto aire de objetividad. Tal característica obliga a los economistas científicos a partir de modelos de equilibrio, no de hechos reales y concretos, pero se agregan variables para aterrizar el modelo. Como consecuencia de aquello ocurrió que mientras más científica se volvió la economía, se volvió más autónoma como disciplina, aquello implicó que la Economía Científica se separó radicalmente de la ética. La economía deja de ser metafísica, no es filosofía, es una ciencia concreta y demostrable. Lionel Robbins, es uno de los pioneros en la tecnificación absoluta de la economía, de hecho define Economía como "la ciencia que estudia la conducta humana como una relación entre fines y medios limitados que tienen diversa aplicación" 6 , y agrega que "[s]e ha sostenido que, porque intenté delimitar claramente el ámbito de la Economía frente a otras ciencias y el de la Economía frente a la filosofía moral, recomendé, por tanto, que el economista se abstuviera de todo interés o actividad fuera de su materia"7.

Importantes economistas, ya no liberales sino que neo-liberales sostuvieron y apoyaron parte de la tesis de Robbins, ya que también creyeron que la economía era una ciencia, pero discreparon en que no tuviera un sustento ético, develaron una posición ética que en la práctica era evidente, no así en la teoría. Sobre eso, es necesario detenerse en las formulaciones de F. A. Hayek.

\footnotetext{
Hopenhayn, Martín. Repensar el trabajo. Historia, profusión y perspectivas de un concepto. Buenos Aires, Argentina: Norma, 2001, p. 97.

5 No es lo mismo que precio. La economía política es aquella que se ocupa del valor. El valor puede traducirse en precio. Es la humanidad que un producto contiene, el trabajo necesario para producir un objeto.

6 Roвbins, Lionel. Ensayo sobre la Naturaleza y Significación de la Ciencia Económica, En: Ramón, José María (comp.). Recortes para la cátedra de Epistemología. Trelew, Argentina: Universidad Nacional de la Patagonia San Juan Bosco, 2009, p. 15.

7 Ibid., p. 2.
} 
Hayek concuerda con Robbins en que la economía es una ciencia pero se aleja de este al plantear que la economía no es una ciencia pura sino que está relacionada con otras áreas de las humanidades, por ejemplo con la filosofía y la ética. En varios de sus artículos y libros plantea que existen ciertos fundamentos éticos de una sociedad libre, por lo que en la teoría hayekiana volvemos a encontrar una relación entre economía y ética. Hayek señala que hay ciertos sentimientos que son propios de las sociedades primitivas y arcaicas, pequeñas sociedades no desarrolladas. Estos sentimientos son la solidaridad y el altruismo y hacen posible la supervivencia de los individuos en colectividades no civilizadas en que la producción debe distribuirse. Las sociedades civilizadas, grandes ordenes espontáneos, han sido capaces de adoptar normas no pensadas, completamente contrarias a los sentimientos espontáneos, normas que permiten por sobre todo la libertad individual. En tal sentido Hayek radicaliza los argumentos y la libertad propuesta por teóricos liberales como Smith o Locke.

El mercado es la institución en que los individuos pueden experimentar la libertad, es ahí donde los individuos son libres ya que pueden relacionarse e intercambiar con individuos extraños buscando su propio beneficio. Cualquier intervención social por parte del Estado es contraria a la libertad.

Como veremos, al adoptar esta posición respecto a la libertad individual y el mercado se suscitan problemas que, por decir lo menos, impiden la libertad de unos para permitir la de otros.

En contraposición a la teoría neoliberal, John Maynard Keynes propone un rol activo y benefactor del Estado, entregando a los individuos ciertas garantías y beneficios, en donde destaca la búsqueda del pleno empleo. La teoría keynesiana busca sacar al capitalismo de las crisis pero no soluciona los problemas centrales del capitalismo. Tal como afirman Paul Sweezy y Paul Baran, el keynesianismo busca salvar al capitalismo de sus crisis cíclicas de sobreproducción haciendo que el Estado intervenga económicamente creando empleos para equilibrar el desbalance. Por otro lado Adela Cortina afirma que "el keynesianismo más buscaba asegura el capitalismo que lograr la igualdad por motivos éticos." ${ }^{8}$

En tal sentido el Estado Benefactor se posiciona como una alternativa al Estado Liberal, que presenta problemas más graves aún, como por ejemplo, que al exacerbar la libertad individual se pierde el sentido de la comunidad. También, al no tener políticas sociales ocurre que algunos individuos poseen más y mejores herramientas para su desarrollo que otros, además de arrojar al juego del mercado derechos fundamentales como la educación y la salud, en consecuencia habrán algunos que por condiciones económicas no podrán acceder a ellos.

Conscientes de tales problemas surge un movimiento liberal que ya no sólo busca asegurar el capitalismo haciéndolo menos salvaje, sino que se preocupa de la desigualdad, este movimiento es denominado Liberalismo igualitario o Igualitarismo liberal. Uno de sus padres y

8 Contina, Adela. Ciudadanos del mundo: Hacia una teoría de la ciudadanía. Madrid, España: Alianza Editorial, 2001, p. 82. 
gran representante de aquello es John Rawls, quien lleva los postulados kantianos que mencionamos (universalización de las normas, ética deontológica y no instrumentalización) ya no solo al ideario de la Libertad sino que también al de la igualdad, en eso se basará la justicia. El gesto de Rawls pretende llevar el kantismo, más allá del plano de la libertad, hasta el plano de la economía y lo social.

El rescate del kantismo, de la deontología y del universalismo que hace Rawls fue, en términos prácticos y teóricos, una fuerte ruptura en la teoría liberal, pero presenta para algunos un problema: es monológico y no dialógico. En tal sentido la ética dialógica de Habermas se hace cargo de aquello.

Habermas logra identificar un gran problema en la ética kantiana: su falta de praxis, ya que no considera de manera efectiva las relaciones entre individuos. Ya "[n]o se trata aquí de universalizar monológicamente y apreciar la contradicción de la máxima con el pensar o el querer: se trata de comprobar la unión de la voluntad particular con la universal a través de un proceso de universalización dialógica; a través del dialogo real entre aquellos que se verán afectados por la norma." Lo que se busca es lograr consenso entre quienes resulten afectados por cualquier acción o norma. En tal sentido el lenguaje juega un rol fundamental ya que hace posible el entendimiento social.

La relación entre ética y economía que puede hacerse desde la teoría habermasiana parte de la distinción entre interacción y trabajo que hace Habermas. Tal separación es una crítica a Marx, quien según Habermas reduce la formación de los individuos al trabajo (acción racional con arreglo a fines) y no considera el proceso social de formación dado por la interacción o la acción comunicativa ${ }^{10}$. El proyecto de Habermas rompe con Marx y abre la posibilidad de crear normas universales bajo un procedimiento dialógico para cualquier ámbito de la vida humana, incluso para la economía y como veremos para las empresas.

\section{LA PROPUESTA DE ADELA CORTINA}

\section{I. Del Estado Liberal y Benefactor, hacia el Estado de Justicia}

Tuvimos que hacer aquella reconstrucción de la relación entre economía y ética ya que para entender el proyecto filosófico de la profesora Adela Cortina es absolutamente necesario. La propuesta ciudadana que propone nuestra autora identifica dos tipos de Estados: el Estado Liberal y el Estado benefactor, pero no se adscribe a ninguno, sino que propone una tercera y nueva forma de estado, el Estado de Justicia.

9 Cortina, Adela. La escuela de Francfort. Madrid, España: Cincel, 1992, p. 155.

10 La enajenación según Habermas es sólo económica. 
Tal y como ya revisamos el Estado Liberal se preocupa de garantizar la libertad individual de los que lo conforman, cualquier intervención social por parte de éste es ilegítima. El mercado es la institución que garantiza tal libertad. Un ejemplo claro de esto, es la propuesta de Milton Friedman en La Libertad de Elegir ${ }^{11}$ en un artículo que se titula ¿Quién protege al trabajador?, Friedman señala que no son ni los sindicatos, ni el Estado los responsables de la protección y de las mejoras de los trabajadores, sino que el mercado, el aumento de productividad beneficia a todos los actores económicos, "este es el modo en el que un sistema de mercado libre distribuye los frutos del progreso económico entre los ciudadanos. Este es el secreto de la enorme mejora de las condiciones de la clase trabajadora a lo largo de los dos últimos siglos"12. El gran problema del Estado Liberal es que se preocupa de entregar libertad a los ciudadanos (de un determinado tipo) y no se pronuncia sobre la igualdad, además de hacer dependientes a los individuos de un mercado al que no le atañe la justicia.

Como señalamos el Estado Liberal presenta graves problemas de exclusión social en que algunos no pueden participar del juego del mercado, por lo que son marginados del sistema. El Estado benefactor pretende solucionar aquellos problemas entregando a los individuos Derechos Sociales, tales como trabajo, educación, salud y previsión, con el objetivo de dar al menos un piso a todos los ciudadanos. El problema del Estado benefactor es que hace a los ciudadanos dependientes de la clase política y de decisiones burocráticas, además de crear ciudadanos pasivos y no activos.

La profesora Adela Cortina propone pasar a un estado de Justicia. Tal Estado debe considerarse un Estado Social de Derecho, un Estado que no lo controle todo sino que garantice mínimos entendidos como mínimos de justicia. El Estado de Justicia apunta tanto a la libertad como a la igualdad, por lo que los ciudadanos serían legítimos propietarios de bienes sociales. "Cada persona debe a la sociedad mucho, tanto de sus facultades como del producto de ellas. Carece, pues, de sentido que los bienes sociales no estén socialmente distribuidos de forma de que cada uno de sus legítimos propietarios goce al menos de un ingreso básico, una vivienda digna, un trabajo, asistencia sanitaria, educación, apoyo en tiempos de vulnerabilidad, amén de esos bienes públicos que no pueden individualizarse"13.

En concordancia con un Estado de Justicia, la profesora Cortina propone una síntesis entre el Liberalismo igualitario y el Comunitarismo, sin olvidar la importancia y el aseguramiento de los Derechos Económicos Sociales y Culturales. En tal proyecto la ciudadanía requiere de dos cosas. 1) Un ideal de justicia racional, preocupada por la igualdad social. Este es el aporte del Liberalismo Igualitario. 2) Un sentimiento de pertenencia a una comunidad. Los comunitaristas argumentaron que el ideario de justicia rompe con el sentimiento de comuni-

\footnotetext{
11 Friedman, Milton y Friedman, Rose. La libertad de elegir. Hacia un nuevo liberalismo económico. Barcelona, España: Grijalbo, 1980.

12 Ibid., p. 342.

13 Cortina, Adela, op. cit. (n.9), pp. 91-92.
} 
dad, de esto que Adela Cortina propone diferenciar entre justicia y vida buena. En tal sentido, la conjunción entre comunidad y justicia, hace posible distintos tipos de ciudadanía, a saber: ciudadanía política, civil, social y multicultural. Para nuestro análisis sólo nos detendremos en la ciudadanía económica

\subsection{Ciudadanía económica}

El concepto de ciudadanía económica ha ido tomando fuerza paulatinamente. Lo que se busca es la participación activa de los ciudadanos en la toma de decisiones económicas ya no como siervos o súbditos sino como señores de sí mismos. La ciudadanía económica es una ciudadanía de los afectados, de todos los afectados, implica tanto una ética discursiva aplicada al ámbito de la economía y la empresa; como un capitalismo de afectados o stakeholder capitalism. El proyecto de la profesora Cortina se hace cargo de ambos ejes.

Antes de continuar es necesario aclarar un par de cosas. Primero, por qué me refiero al proyecto de Adela Cortina. Creo que la propuesta de la profesora valenciana es completa e innovadora, además de poner en jaque ciertas instituciones y prácticas en pos de un proyecto de ciudadanía, y de romper con los anteriores intentos de ética empresarial existentes. En segundo lugar es necesario aclarar que el plan de trabajo, desde ahora en adelante, es avocarnos a la idea que Cortina propone en su libro Ética de la empresa ${ }^{14}$ : que para lograr verdadera ciudadanía económica es necesaria una ética empresarial, enmarcada en una ética aplicada y cívica. Por lo que, lo primero que debemos hacer, es explicar el concepto de ética aplicada.

\subsection{LA ÉTICA APLICADA}

Ricardo Maliandi distingue cuatro niveles de reflexión, a saber:

a) En primer lugar la reflexión moral. Este nivel dice relación con las discrepancias sobre el actuar que se puede tener con otro, o con las posibilidades de acción respecto a un determinado problema moral.

b) El segundo nivel corresponde a la ética normativa. Está estrechamente relacionado con el nivel anterior, se relaciona con "la búsqueda de fundamentos de las normas y la crítica de aquellas normas que no nos parecen suficientemente fundamentadas o incorrectas"15.

c) En tercer lugar Maliandi identifica el nivel de la metaética. El que dice relación con el lenguaje, es "un tipo de reflexión que analiza el significado y uso de los términos morales" se evalúa la calidad y coherencia de los principios que se han pensado en el segundo nivel.

14 Cortina, Adela. Ética de la empresa. Claves para una nueva cultura empresarial. Madrid, España: Trotta, 2000.

15 Maliandi, Ricardo. Ética: conceptos y problemas. Buenos Aires, Argentina: Biblos, 2004. p 48. 
d) El cuarto y último nivel reconocido es la ética descriptiva. Este consiste en observar el fenómeno moral desde una posición apartada, o más bien como un tercero.

Hago referencia a los niveles de reflexión planteados por Maliandi, debido a que para responder a la pregunta ¿qué es la ética aplicada?, es necesario explicarlos y tenerlos en cuenta. El término ética aplicada puede sonar extrańo ya que la ética es práctica en si misma. Entonces, ¿por qué usamos el término ética aplicada? ¿En qué consiste la aplicabilidad? Ocurre que en la ética aplicada podemos distinguir dos niveles de reflexión actuando conjuntamente, a saber: la reflexión moral y la ética normativa. El primer nivel dice relación, entre otras cosas, con el ¿qué debo hacer?, es decir es práctico en si mismo, "es ya una reflexión "aplicadora" de normas"16. El problema surge cuando nos referimos al segundo nivel, es decir, a la ética normativa, debido a que esta "no se ocupa de aplicar las normas sino de determinar cómo y cuándo esa aplicación es 'válida’17.

La relación entre la reflexión moral y la ética normativa puede entenderse como la relación entre lo pertinente a la razón (la ética normativa) y la acción (reflexión moral). Es en este sentido que la ética aplicada es tal, donde la ética normativa es aplicada por la reflexión moral, por lo que la conexión entre aquella (la ética normativa) y el problema es indirecta. La ética (el por qué de la reflexión) es aplicada a la moral (el qué de la reflexión).

En estos términos la ética empresarial es ética aplicada, al buscar aplicar cierta normatividad al actuar de las empresas como organizaciones

\subsection{La Ética empresarial}

La ética es saber, un saber práctico, no es conocimiento vago y meramente teórico que apunta a su enseńanza en las escuelas, sino que está pensado para la acción. De esta manera tanto la ética en general, como la ética aplicada en particular, surgen como posibilidad de dos cosas, en primer lugar de la vida en sociedad y en segundo, de la libertad. Lo que las teorías éticas buscan es que sea posible la convivencia entre hombres distintos.

Es precisamente ese el objetivo de la ética cívica. La profesora Adela Cortina expresa que hace posible "la convivencia entre ciudadanos que profesan distintas concepciones religiosas, ateas o agnósticas, siempre que compartan unos valores y unas normas mínimas"18. Ana Escribar W. señala que: "como consecuencia del proceso de globalización en curso hoy conviven dentro de una misma sociedad los que podríamos considerar como "extrańos morales"; esto es,

\footnotetext{
6 Ibid., p. 64.

17 Ibid., p. 68.

18 Contina, Adela, op. cit. (n.15), p. 37
} 
personas que tienen distintas concepciones de la vida buena, distintos ideales en lo que respecta al bien y la felicidad, distintas creencias religiosas, distintas ideas políticas y filosóficas (...) y que (...) demanda perentoriamente condiciones de igualdad"19.

Como se expresa tanto en las obras de Cortina y Escribar, lo que hace posible la convivencia en una sociedad de por sí heterogénea, son los mínimos morales, es decir ciertas normas que son vinculantes para todos los ciudadanos, sin excepción. Lo que se busca nos es encontrar un sólo proyecto de sociedad o de felicidad, sino ciertas normas de carácter innegociable e irrenunciable, que no sean impuestas sino a las que se llegue de motu proprio.

Los mínimos morales son la base de la ética cívica, y del pluralismo moral, los que hacen posible además de la convivencia, la diferenciación, o sea, la búsqueda de nuestros propios fines, respetando la autonomía del otro y, obviamente los mínimos.

Para la profesora Cortina, la ética cívica es en primer lugar una ética de mínimos, pero también una ética de ciudadanos, no de súbditos, no es la ciudadanía hobbesiana en que el soberano decide lo que es mejor para sus súbditos, la justicia no es impuesta. En tercer lugar la ética cívica es una ética de la modernidad, una ética ilustrada, en el sentido kantiano, donde los ciudadanos son capaces de autodeterminarse haciendo uso su propia razón.

En resumidas cuentas la ética cívica y la ética aplicada van de la mano. La primera busca hacer posible la convivencia de los hombres en tanto ciudadanos, busca el respeto entre estos y su autonomía. La segunda busca crear la conexión entre el ejercicio de una profesión o disciplina y la ética normativa, y llevar a la práctica el fruto de esa conexión. Para lograr su objetivo la ética aplicada debe tener en cuenta a la ética cívica. Por otro lado, la ética cívica es posible gracias, -entre otras cosas-, a la ética aplicada.

Ya aclarados los términos en los que me referiré a la ética de la empresa, me es posible aclarar en qué sentido la ética de la empresa puede entenderse como una ética aplicada y una ética cívica. En primer lugar, la ética de la empresa es una ética aplicada porque busca llevar a la práctica empresarial elementos que son recogidos de varias teorías éticas normativas ${ }^{20}$. El punto es que una empresa es una organización que lo que busca es la satisfacción de las necesidades humanas, pero ¿̇a qué precio?, ¿respetando cuáles normas? La ética empresarial es ética aplicada ya busca limitar el actuar y las relaciones tanto interiores como exteriores de una empresa desde la elementos normativos de teorías éticas. En segundo lugar es una ética cívica, ya que además busca que quienes forman parte de la empresa sean tratados como ciudadanos que puedan lograr su autonomía. "El problema de la ética empresarial es cómo combinar eficiencia económi-

19 Escribar W., Ana. "La ética aplicada, sus condiciones de posibilidad y exigencias a las que responde", en: Revista de Filosofia, No 60, pp. 19-28, Santiago, Chile: Departamento de Filosofía Facultad de Filosofía y Humanidades, Universidad de Chile, 2004, p. 20.

20 En la próxima sección señalaremos cuales. 
ca con libertad individual, equidad social y respeto por los valores absolutos" 21 . De esta manera la ética de la empresa no es la aplicación de normas preestablecidas, con el objetivo de enmarcarse dentro de una determinada conducta, tampoco consiste sólo en la mera aplicación de los principios generales ya preestablecidos, sino que se pretende averiguar cuáles son los bienes internos que la actividad empresarial puede y debe proporcionar a la sociedad toda, encontrar metas a perseguir además de valores y hábitos para incorporar. En concordancia con lo anterior, lo que caracteriza a la ética de la empresa es un carácter sistemático y evolutivo, y no atiende a un catálogo de normas al que deba ceñirse.

¿Cómo surge la ética empresarial? Ya Aristóteles se refería con recelo y desaprobación a la cremástica, pero poco a poco, el deseo de acumulación fue aceptándose, hasta que, como señalamos, la teoría liberal hizo una defensa ética de tal deseo ${ }^{22}$.

A medida que pasaban los años el capitalismo fue mutando sobre todo en sus técnicas de producción. Son importantes a este respecto el paso del taylorismo al fordismo o postaylorismo y al actual posfordismo. Nos parece, tal y como se apuntan en varias obras sobre ética de la empresa, que la década de los setenta es crucial en el desarrollo del tema, ya que es en este periodo donde surge la ética de la empresa como una ética aplicada. También por la necesidad de crear capital social que puede ser definido como la relación que la empresa forja con la sociedad civil, relación basada en la confianza mutua., como complemento al capital físico - maquinas, edificio, tierras - y al capital humano - trabajadores y técnicas de producción-.

Otro punto que es interesante hacer notar es que "el fin de las ideologias" favoreció el nuevo surgimiento de la ética empresarial, y no sólo de ella, sino del conjunto de los que ha dado en llamarse <<éticas aplicadas > ... [Además de] el interés por las buenas prácticas en la economía y la empresa" 23 .

Existieron proyectos, como el de Milton Friedman, en que se establecía que una empresa ética era aquella que aumentaba las utilidades de sus accionistas. Pero debido a factores como la doctrina social de la iglesia o los movimientos contraculturales en respuesta a lo que ocurría en Vietnam, la sociedad civil comenzó a mostrar su descontento con los grandes empresarios y a mostrar su rechazo a la falta de escrúpulos con los que actuaban. En respuesta a esto surgieron proyectos como el de la Harvard Business Review en que se buscaba incentivar la formación de líderes fuertes tanto en conocimiento como en formación ética

Los proyectos siguen siendo insuficientes por lo que en la década de los noventa nace el proyecto de Adela Cortina. Este proyecto lo que se busca es la formación de empresas excelentes,

\footnotetext{
21 Martínez, Horacio. La ética empresarial al comienzo del nuevo milenio: una aproximación bibliográfica y pedagógica. Bogotá, Colombia: Pontificia Universidad Javeriana, 2005, p 8.

22 Incluso Jeremy Bentham llegó a escribir En defensa de la usura.

23 Cortina, Adela. op. cit. (n.2), pp. 27-28.
} 
interesadas ya no en la mera eficiencia y utilidad económica sino que en la calidad de los productos y servicios. Es de acuerdo a esto, que la ética de la empresa se perfila en el marco de una ética civica. La preocupación de la empresa ya no sólo se restringe a su propia individualidad sino que se extiende a todos los stakeholders, es decir a todos aquellos sujetos afectados y relacionados a la producción y al consumo.

La ética de la empresa como ética aplicada y ética civica, recoge elementos de distintas teorías éticas normativas, y es precisamente desde ahí donde surge su aplicabilidad. Lo que se busca hacer es compatibilizar elementos de teorías diversas para posibilitar que el actuar empresarial se condiga con el actuar ético.

Antes de entrar de lleno en el asunto, debemos señalar es que la ética de la empresa basa gran parte de su sustento filosófico en la ética del discurso o dialógica, pero además recoge elementos de distintas tradiciones que apuntan a distintos tipos de racionalidades. Lo que se busca es tratar de recoger lo mejor de estos para aplicarlos en el marco de una ética ciudadana.

En primer lugar, recoge elementos de la racionalidad prudencial, vinculada a la tradición aristotélica. Principalmente esta tradición se caracteriza por la edudaimonía, o por considerar a la felicidad como el fin último de la humanidad, se debe considerar también la prudencia en los actos y el carácter casuístico de su análisis. Sobre todo debe destacarse la importancia que en esta teoría juegan las virtudes.

La segunda tradición corresponde al utilitarismo vinculado a la racionalidad calculadora. Su base está en el principio de maximización de la felicidad, entendida como placer, en este sentido las acciones se miden por sus consecuencias. Se busca la felicidad para el mayor número de personas.

Basada en el pensamiento de Kant surge la tercera tradición (kantiana), de la que nace la racionalidad práctica. Lo que caracteriza a esta tradición es el respeto por todos los seres humanos por ser tales, nadie puede ser un medio sino siempre debe ser un fin en si mismo. Es importante recalcar que bajo los principios de esta tradición se erige una de las fundamentaciones más importantes de los Derechos Humanos.

Por último y como ya he señalado la ética de la empresa recoge elementos de la tradición dialógica, de la que surge la racionalidad comunicativa. Gracias al aporte de esta teoría pueden consensuarse los llamados minimos morales. Lo que se busca es que se satisfagan intereses universales y no particulares.

Josep Lozano propone una hermenéutica de la responsabilidad, la que "surge de una comprensión integrada e integradora de las tradiciones utilitaristas, aretológica y deontológica, realizada desde la problemática de la BE [o Business Ethics] y en diálogo con ésta"24.

24 Lozano, Josep. Ética y empresa. Madrid, Espańa: Trotta, 1999, p. 28. 
Debemos ser bastante cuidadosos respecto a lo anterior, ya que lo que se busca no es hacer una mezcolanza de teorías éticas normativas y presentar un proyecto filosófico, sino lo que se pretende es rescatar elementos de cada una de esas teorías que en definitiva, hagan posible los minimos intransables de acción. Lo que hace posible la convergencia de todos en aquellos mínimos es precisamente la racionalidad comunicativa.

La tradición dialógica se sirve en gran parte de los postulados éticos de Inmanuel Kant, (entre estos los de universalidad de las normas y de la no instrumentalización del prójimo) pero a diferencia de éste, plantea que la racionalidad moral se obtiene dialógicamente y no en una relación del sujeto consigo mismo.

El discurso o diálogo hace posible el consenso sobre normas morales de carácter universal. En ese sentido la racionalidad comunicativa llevada a cabo en el dialogo hace posible el acuerdo de tales mínimos. Una condición sine qua non para el discurso es que todos los interlocutores sean considerados interlocutores validos, es decir que todos aquellos con capacidades de discursivas puede participar del dialogo. Se busca una relación lo más cercana a la simetría. El consenso a través del dialogo da respuesta a una pregunta fundamental ¿̇de que es digno el ser humano? "la ética discursiva es, en principio, deontológica, porque no se ocupa directamente de la felicidad ni de las consecuencias, sino de mostrar cómo la razón humana sí ofrece un procedimiento para decir qué normas son moralmente correctas: entablar un diálogo entre todos los afectados por ellas que culmine en un acuerdo, no motivado por razones externas al dialogo mismo, sino porque todos están convencidos de la racionalidad de la solución.” 25

La ética dialógica, no busca terminar como las tradiciones antes nombradas, sino que es buscar en ellas la base de los mínimos.

En resumen, según lo que ya hemos dicho hasta ahora, desde que existe el capitalismo la ética, la economía y la empresa se han relacionado de distintas formas. La ética de la empresa como ética aplicada, cívica, y aunque suene extrańo, deontológicamente discursiva, es una muestra más de la posible relación existente.

¿Cuáles son los motivos para el nacimiento de la ética empresarial? Casos como el de Watergate ${ }^{26}$ hicieron entre otras cosas que la gente perdiera la confianza en las empresas, por lo que se hizo una necesidad de primer nivel para estas recuperar lo perdido. En un primer momento el fundamento fue que la confianza y credibilidad en las empresas es rentable.

Recuperar la confianza en las empresas es una tarea de largo plazo, en el que se debe poner en tela de juicio el funcionamiento de su totalidad. En este marco se encuadra la Responsabilidad Social Empresarial. Esta consiste en que las empresas reconocen una obligación de carác-

25 Cortina, Adela, op. cit. (n.15), p.31.

26 Escándalo sobre espionaje ocurrido en Estado Unidos en 1972, involucrado el ex presidente de los Estados Unidos, Richard Nixon. 
ter moral para con los consumidores y la sociedad toda. Las organizaciones entienden su posición en la sociedad y adoptan un compromiso con ésta, con el objetivo de contribuir al mejoramiento social, cultural y ambiental. En este sentido, resaltan las figuras del manager y del directorio, que son la cara visible de las empresas. Se busca que el compromiso ético de las empresas no esté basado exclusivamente en la utilidad que para las organizaciones pueda entregar la ética, sino que en un verdadero compromiso cívico. Independientemente de que de todos modos en una sociedad preocupada por el medioambiente y por las diferencias sociales y culturales el actuar ético en una empresa puede ser, querámoslo o no, una estrategia comercial fuerte ${ }^{27}$.

Pero para Cortina, "la ética de la empresa no es una moda, no es una cosmética [sino que debe entenderse como] una autentica necesidad social. ${ }^{28}$

Ya claro el fundamento histórico y teórico nos ocuparemos de explicar cuál es el objetivo de acción de la ética empresarial propuesta por Cortina, qué es lo que busca lograr o recuperar y cómo busca hacerlo. ¿Cómo llevarla a cabo? En primer lugar la ética de la empresa debe entenderse como "un modo de resolver moralmente conflictos de acción". En concordancia con lo que he señalado anteriormente, la ética empresarial, al ser aplicada y civica lo que busca que es que ante conflictos morales se puedan encontrar soluciones a estos a través de un procedimiento de argumentación, un proceso dialógico. Nuevamente insisto en que la ética de la empresa (propuesta por Cortina) no busca un decálogo de normas, sino que las normas se construyen por consenso.

La ética de la empresa no sólo debe entenderse como una ética del trabajo, o como ética en la empresa, sino que debe ocuparse tanto de las relaciones internas, las relaciones que involucran tanto a trabajadores como a directivos; y las relaciones externas, con sus consumidores, proveedores, con el medio ambiente y la ciudadanía en general.

Lo que se busca es aplicar principios éticos normativos a la práctica de los negocios y las prácticas empresariales en general. Esta podría identificarse como una primera fase, pero tal y como apunta Adela Cortina, la "transición a una segunda fase se produce cuando, a través de la consideración moral de la acción de los directivos, se percatan de que lo son de organizaciones: las organizaciones tienen obligaciones sociales que trascienden sus funciones económicas"29. Una tercera fase consiste en "reconstruir la legitimidad de la organización y para ello es necesario comprender la organización como proceso organizativo, poner énfasis en los proceso de

\footnotetext{
27 Casos como el 1x1 (compre uno y regalamos uno, por ejemplo, a un niño en África), o el uso de materiales orgánicos son buenos ejemplos para ilustrar el asunto.

28 Cortina, Adela, op. cit. (n.15), p. 85.

29 Ibid., p. 87.
} 
toma de decisión, y en la interrelación del conjunto de elementos que configuran el proceso de constitución de la organización y la comprensión de sus finalidades como clave para comprender los procesos de toma de decisión"30.

Para esto es necesario la formación ética de quienes deben llevar esto a cabo, en otras palabras se busca educar en la ética empresarial (como ética cívica) a los directivos de la empresa, especialmente a aquellos a cargo o que trabajan en recursos humanos.

Aquella empresa alta de moral ${ }^{31}$ no solo debe preocuparse de producción más y de las utilidades, sino que debe apuntar a producir con calidad y debe comprender de que sus relaciones son con seres humanos, en este sentido se debe recordar el principio de respeto por la humanidad postulado por Kant.

La ética empresarial permite entre otras cosas hacer diferencia en el mercado. Una empresa alta de moral resalta por sobre sus competidoras debido a su actuar no-instrumental. Esto permite, como consecuencia, que las empresas éticas sean del todo rentables "porque reduce costes de coordinación externos e internos en la empresa: posibilita la identificación con la corporación y una motivación eficiente."32

Llegamos a un punto clave en nuestro análisis, el de dotar de contenido a la ciudadanía económica entendida en estos términos. El proyecto de Adela Cortina es crítico del capitalismo, pero no busca deshacerse de él, sí modificarlo desde una perspectiva ética, en tal sentido, el mercado ya no es un fin sino que un medio para el logro de este proyecto.

Hay cuatro puntos a los que, desde la perspectiva de Cortina, una empresa necesariamente debe atender para crear ciudadanía económica: 1) a metas sociales; 2) a mecanismos adecuados para alcanzarlas, usando el mercado, ahora no como fin, sino que como medio; 3) al marco jurídico político vigente (atendiendo a derechos políticos, civiles y sociales; 4) a las críticas de la conciencia moral crítica, ejemplo de esto es la crítica a la precarización de las relaciones laborales.

Me parece que he expuesto lo fundamental sobre la ética empresarial. Lo expuesto, hasta ahora, hace posible una comprensión, a lo menos general del plan y posibilita entender en qué sentido la ética empresarial apunta a una ciudadanía económica. 


\section{CRÍTICAS PARA LA CONTRIBUCIÓN A UN PROYECTO DE PARTICIPACION}

Creo que el aporte de Adela Cortina es clave y limita bastante el actuar del capitalismo que hoy por hoy es despiadado, especialmente en nuestro país. Nuevamente, tal y como lo hizo el liberalismo igualitario, se busca compatibilizar igualdad y libertad, el gesto teórico es bastante favorable a todos en términos ciudadanos, es en definitiva un reproche a la sociedad neoliberal. Pero me parece que la propuesta de la profesora Cortina prescinde de algunos detalles que me gustaría señalar. De este modo nuestra primera crítica apunta al consenso dialógico que propone para alcanzar las metas, en contraposición a aquello propongo reconocimiento. En concordancia con esto me parece que es posible apuntar a la participación económica radical, para el logro de una economía más allá de las fronteras del mercado.

La primera crítica contrapone el reconocimiento hegeliano (explicado desde Honneth), al dialogo habermasiano propuesto por Cortina. El punto que queremos defender es que crear ciudadanía desde el consenso dialógico supone interlocutores válidos, lo que me parece, a lo menos, dudoso. Para poder entender la crítica, es necesario detallar algunas cosas sobre la teoría del reconocimiento

\section{I. LA LUCHA POR EL RECONOCIMIENTO}

La teoría del reconocimiento debe entenderse en el marco de la Teoría Crítica, en la que Axel Honneth se presenta como quizás el más importante representante de su ya tercera generación. Honneth lo que busca no es precisamente mostrarse como un continuador de esta sino más bien como un crítico.

En el contexto de la desregulación desenfrenada del capitalismo bajo la teoría neo-liberal surgen, lo que podríamos llamar, preocupaciones sociales. El producto de este fenómeno es, en palabras de Honneth, "un aumento en la sensibilidad moral: [personas] conscientes, gracias a toda una serie de nuevos movimientos sociales, del valor político de la experiencia del menosprecio social y cultural." 33 Desde esa óptica debe entenderse la libertad como no-dominación, en la que el reconocimiento juega un rol fundamental par su logro.

La concepción sobre el concepto de reconocimiento que propone Honneth es heredera de manera directa de la teoría hegeliana del reconocimiento, que va más allá del respeto de la humanidad como fin de Kant, y de la propuesta de Fichte "de que los individuos podían adquirir consciencia de su libertad sólo si se incitaban mutuamente a hacer uso de su autonomía y, en consecuencia, se reconocían los unos a los otros como libres." 34

33 Honneth, Axel. Reconocimiento y menosprecio. Buenos Aires, Argentina: Katz, 2010, p. 12.

34 Ibid., pp. 18-19. 
La teoría del reconocimiento no debe entenderse como sinónimo de las políticas de identidad relacionadas al multiculturalismo, sino que debe entenderse en el marco de las luchas sociales, en este caso en una lucha por el reconocimiento, no es sólo el respeto por la diferencia.

Según el actual director de del Instituto de Investigación de la Universidad de Frankfurt, debemos entender el reconocimiento "como una reacción comportamental con la cual respondemos racionalmente a cualidades evaluativas que aprendimos a percibir en la medida que estamos integrados en la segunda naturaleza de nuestro mundo de vida" 35 , de esta forma, si entendemos el concepto de tal manera, "el término de reconocimiento pasa a designar un comportamiento reactivo [no meramente pasivo] con el cual respondemos a las propiedades de valor de otras personas de forma racional."36

Axel Honneth es un gran crítico de la ética del discurso habermasiana, la teoría del reconocimiento puede entenderse como una crítica a la primera, aunque toma elementos de aquella. Un elemento importante, en las que ambas se unen, es que los dos proyectos se encuadran en la búsqueda de la Justicia.

En el libro La Lucha por el Reconocimiento (1997) y en varias de sus obras, Honneth distingue tres tipos de humillación o menosprecio, la figura opuesta al reconocimiento, en términos hegelianos la negación del reconocimiento, a saber: la agresión, la desposesión de derechos y la exclusión social. Por lo que en contraposición existen tres formas de reconocimiento: el amor, el derecho y la solidaridad entendida como valoración social.

El amor es el reconocimiento en el marco de la familia y entre amigos (amor como amistad) y que genera en los individuos mutuamente reconocidos autoconfianza.

El derecho como reconocimiento consiste en que todos los individuos pertenecientes a una determinada sociedad, tengan o sean poseedores de los mismos derechos pero además que tengan la posibilidad de ejercer y exigir aquellos derechos. La privación de derechos como humillación se puede traducir en el no reconocimiento como persona -a ahora jurídica- del sujeto humillado. Es importante recalcar que la teoría de Honneth no apunta a un reconocimiento jurídico sólo de Derechos Civiles y Políticos, sino que los Derechos Sociales juegan un rol fundamental en su teoría. En tal sentido esta segunda forma elemental de reconocimiento esta estrechamente ligada a las luchas históricas, ya que a través de ellas es posible la obtención de aquel reconocimiento. Así, los Derechos Sociales para Honneth no están fundamentados en

35 Honneth, Axel. "Grounding recognition: a rejoinder to critical questions", en: Inquiry, Vol. 45, no 4 (dic. 2002), pp. 499512. Londres, Reino Unido: Taylor \& Francis Group, 2002, p. 513.

36 Mezquita, Miriam. "Introducción a la Crítica del Agravio Moral”, en: Honneth, Axel. Crítica del Agravio Moral. Patologías de la sociedad contemporánea. Buenos Aires, Argentina: Fondo de Cultura Económica, 2009, pp. 37-38. 
una intrinseca dignidad humana, sino que en los logros históricos del pueblo. "La actitud positiva que un individuo puede adoptar hacia sí mismo cuando experimenta ese tipo de reconocimiento jurídico es la del autorespeto elemental." "37

La tercera forma de reconocimiento corresponde a "una relación de aprobación solidaria y apreciación de las capacidades y formas de vida desarrolladas individualmente" 38 , recordando el tinte hegeliano del autor, Paul Ricoeur seńala que este tercer tipo de reconocimiento "se toma como término de referencia, en toda su amplitud, el concepto hegeliano de "eticidad": es en este plano en el que la "vida ética" se muestra irreductible a los vínculos jurídicos"39. En definitiva esta tercera forma es una valoración social de los individuos por su contribución a la sociedad, este reconocimiento lleva al individuo a la autoestima. Lo último que debe señalarse respecto de este tercer tipo de reconocimiento es que también puede ser entendido como una "comunidad de valores compartido" 40 , este tipo de reconocimiento es además, aprobación social.

Nos parece que hemos expuesto lo central sobre la obra de Honneth ${ }^{41}$, que sirve para nuestro análisis por lo que podemos entrar de lleno en la primera parte de nuestra crítica. Al ser el Derecho la segunda forma de reconocimiento, nos gustaría señalar algunas cosas sobre esto. La ética de la empresa no se ocupa del Derecho o va más allá de él, de hecho podríamos entenderla como un complemento a lo establecido por el sistema jurídico. Pero hay casos en que la ética de la empresa no puede ser suficiente ya que la base jurídica es exigua, debido a que hay normas que siempre impedirán el reconocimiento. En relación con esto el caso chileno puede ser bastante ilustrativo.

Nuestra Constitución Política, ideada en la dictadura de Pinochet por una comisión sin inclusión popular donde la figura de Jaime Guzmán tuvo bastante relevancia, establece como Derecho Fundamental la igualdad ante la ley. A primera vista cumple con el requisito de la forma de reconocimiento que hace posible el autorespeto, pero nos parece que debemos ir más allá en el análisis. La igualdad ante la ley que otorga nuestra constitución es la igualdad ante un Derecho que no favorece a todos por igual. Ejemplos de esto son: establecer como derecho fundamental la Libertad de empresa apoyada con un rol subsidiario del Estado (en desmedro de un rol solidario), reconocer un derecho de propiedad ultra-protegido y establecer como base institucional la soberanía en la nación y no en el pueblo. Por lo que a nuestro juicio aquella igualdad es ficticia, ya que se encuadra en un Derecho que no da la posibilidad de reconocimiento de todos y no encuadra con las luchas sociales, en definitiva con la historia (en términos hegelianos), además de ser notoriamente excluyente.

\footnotetext{
Honneth, Axel, op. cit. (n.35), p. 27.

Ibid., p. 28.

39 Ricoeur, Paul. Caminos del reconocimiento. Tres estudios. D. F., México: Fondo de Cultura Económica, 2006, p. 256

40 Mezquita, Miriam, op. cit. (n.38), p. 25.

41 Sería irresponsable de nuestra parte si sostuviéramos que hemos resumido o que hemos abordado todas las aristas de la obra de Honneth, por lo que para mayor profundización sobre el asunto recomiendo ir directamente a sus obras.
} 
Me parece que un verdadero reconocimiento jurídico es aquel que considera derechos políticos, civiles y sociales, y estos últimos no quedan sometidos a los otros dos. Se debe garantizar, la educación, la salud, la previsión, el trabajo, entre otras cosas. Además, nos parece que se reconocer como derecho social un ingreso básico universal al estilo de Philippe Van Parijs, el que no debe entenderse equivalente a un salario, es poner en jaque la economía de mercado. La profesora Cortina ha señalado en su obra estar de acuerdo con esto.

Esto garantiza un piso jurídico que pone en jaque la lógica del capitalismo, en tal sentido atacamos el Estado de Derecho de clase.

Me referiré ahora a la tercera forma de reconocimiento. Marx en gran parte de sus obras se ocupó de dejar en claro que el capitalismo pone al modo de producción por sobre los factores de producción. En otras palabras, lo que se busca es mantener y reproducir la forma de producción capitalistas en detrimento de la fuerza de trabajo ${ }^{42}$. Mi crítica surge desde ahí, ya que tal y como señala la profesora Cortina la ética empresarial se enmarca dentro de la ética cívica y ética aplicada, donde lo que se busca es aplicar mínimos morales que no son transables y de los que no se puede disponer. Como he señalado para el logro de estos mínimos es necesario que todos los participantes de este diálogo sean interlocutores válidos. Me parece que precisamente es ese el problema. Un interlocutor válido al considerarse que posee capacidades discursivas debemos entender que está en posición de poder dialogar, se busca que exista algo similar a la simetría entre los interlocutores.

Nos parece que los trabajadores al estar siempre sometidos al sistema capitalista no están en posición de poder dialogar por razones simples: a) por la naturaleza misma de la relación entre el modo de producción y las fuerzas de trabajo; b) por la actual precarización de las relaciones laborales en pos de la eficiencia en la producción: c) debido a que, como señala Honneth, "hay un número creciente de personas que a causa de un desempleo ya no simplemente coyuntural, sino estructural, carecen de la posibilidad de obtener, gracias a las capacidades adquiridas en el proceso de socialización, aquel tipo de reconocimiento llamado apreciación social"ł3; y d) que a nuestro juicio es lo más importante, el mercado capitalista, no sólo económico, basa su lógica en la desvalorización de un sujeto para la valorización de otro.

Mi propuesta no busca que los trabajadores, consumidores y la sociedad en general respeten la diferencia del otro y puedan identificarse en los mínimos, aplicados por aquella empresa alta de moral en su actuar, sino lo que busca un reconocimiento verdadero, el que no apunta a que las empresas actúen de acuerdo a la ética, sino que va más allá.

\footnotetext{
42 A quienes les interese el tema se aconseja revisar el primer tomo de El Capital, en especial sus secciones tercera, cuarta y quinta; también Salario, Precio y Ganancia; la Contribución a la Crítica de la Economía Politica y los Manuscritos Económicos y Filosóficos de 1844.

43 Honneth, Axel, op. cit. (n.35), p. 44.
} 
El verdadero reconocimiento, que haría posible la apreciación social, es aquel que considera el problema de la distribución. "En este sentido la misma lucha por la distribución... se haya anclada en una lucha por el reconocimiento: representa un conflicto alrededor de las jerarquías de valores socialmente institucionalizadas que regulan que grupo social tiene derecho a exigir legítimamente" ${ }^{44}$. Además se debe terminar con reconocimientos que ocultan la humillación, que en cierto sentido pueden considerarse auto-reificantes, reconocimientos ideológicos. Un ejemplo de esto, es aquel en el que se busca ocultar la precarización de las relaciones laborales refiriéndose a los trabajadores como "empresarios de su propia fuerza de trabajo" 45

En resumidas cuentas el problema de la ética empresarial, al basarse en el la ética del discurso, es que no permite el reconociendo verdadero de los individuos, ya que en cierto sentido prescinde de éste. Honneth señala que la ética discursiva omite la etapa del reconocimiento que haría posible un diálogo simétrico (si es que este es posible). En tal sentido el discurso no es dialógico en estricto rigor, ya que de haber dialogo este se produce entre sujetos asimétrico donde uno tiene mejores capacidades y/o posibilidades discursivas que el otro. Lo que quiero expresar con esto es que la propuesta de una salida ética no termina con un problema esencial, el del mercado capitalista, si bien indica que busca ponerlo como medio y no como fin, lo mantiene de igual manera. Mantener el mercado implica la desvalorización de unos para la valorización de otros, que es el argumento de Marx especialmente en el trabajo enajenado.

\subsection{Propuesta de Participación Económica Radical}

Lo que propongo es que para que exista verdadero reconocimiento ${ }^{46}$ es necesario en primer lugar, que se termine la apropiación individual de plusvalía, reemplazada por un aprovechamiento social: nadie debe apropiarse de un trabajo que no le pertenece. Un primer paso son las medidas a las que nos referimos cuando hablamos del ámbito jurídico. Un segundo paso es crear participación económica radical, que rompe con el capitalismo, y con el mercado por la simple razón de que éste "es incompatible con la justicia porque impide la igualdad de acceso a los recursos, es incompatible con los principios de la democracia porque supone una asimetría de poder, y es insostenible en términos ecológicos a causa de su dinámica acumulativa y destructiva" ${ }^{7}$

La forma de participación económica que propongo recoge proposiciones de las obras de renombrados críticos actuales del capitalismo, tales como Alex Callinicos, Michael Albert, Ro-

\footnotetext{
Ibid., p. 43.

45 Mezquita, Miriam, op. cit. (n.38), p. 40.

46 Uso la propuesta de Honneth sólo como un punto de partida, ya que explica de manera bastante concisa la teoría del reconocimiento. No busco servirme de su teoría más allá de eso. Lo que propongo es mucho más radical.

47 Lizárraga, Fernando. "Utopías posibles más allá del mercado", en: Otros Logos. Revista de estudios críticos. Año 1, Número 1, Centro de Estudios y Actualización en Pensamiento Político, Decolonialidad e Interculturalidad. Neuquén, Argentina: Universidad Nacional del Comahue, 2010, p. 76.
} 
bin Hahnel o Pat Devine, entre otros. Debo dejar en claro que no es una puesta en marcha de sus teorías como una receta, sino solo busco proponer elementos y mostrar que otra economía y otro mundo son posibles.

"Desde luego que no se trata de diseñar sociedades de fantasía, donde cada ínfimo detalle ha sido considerado hasta la exasperación; ni tiene mucho sentido embarcarse en la elaboración de ingeniosos experimentos mentales a menos que se tome nota de los movimientos emancipatorios realmente existentes. Se trata, en todo caso, de recurrir a lo que Alex Callinicos ha definido como imaginación utópica, esa "capacidad para anticipar, al menos como bosquejo, una forma de coordinación económica eficiente, democrática y ajena al mercado" ${ }^{48}$. Es una propuesta revolucionaria en el sentido de que rompe con la lógica de las instituciones y con las instituciones mismas. Es una propuesta que es utópica en ya que no tiene lugar o un lugar hoy, ademar rompe con la necesariedad (en el sentido de que algo ocurrirá necesariamente) y da paso a la posibilidad, en tal sentido esta es otra economía y otro mundo son posibles.

"El procedimiento que existe en la actualidad [...] es el sistema de mercados. El procedimiento que solía existir no hace mucho tiempo en la Unión Soviética se denominaba planificación central. La economía participativa rechaza tanto el sistema de mercados como la planificación central y propone en cambio una "planificación participativa"”49. En tal sentido, el proyecto no es un socialismo de mercado al estilo de Dieterich.

Podemos buscar la coordinación negociada de Devine o las parecon de Albert, terminar con la división social del trabajo y pasar a una división funcional de éste, así las tareas no estarían determinadas e institucionalizadas; disminuir las jornadas de trabajo, distinguir entre trabajo libre y productivo (muchos trabajos actuales serían trabajos libres) donde todos tengamos que participar de aquella distinción.

La idea es que la producción sea coordinada entre trabajadores y consumidores, no busco definir como (por lo menos en este trabajo).

Lo que se busca es que en términos de valor nadie sea más que otro, que no hayan clases, no se apunta a un igualitarismo radical en que todos vistan, coman y tengan lo mismo, sino que se busca la Libertad de los individuos en tanto seres humanos, y para eso es necesaria la igualdad en los términos que estamos planteando, en que nadie sea desvalorizado para valorizar a otro. En definitiva se busca armonizar participación en contraposición a la dictadura del mercado, eficiencia en la producción y distribución en contraposición a la absurda sobre producción capitalista; sustentabilidad en contraposición al daño ambiental.

48 Callinicos, Alex. Igualdad. Madrid, España: Siglo Veintiuno, 2003, p. 160.

49 Albert, Michael. Economía participativa. Conferencia en Woods Hole. Ciudad, Estados Unidos: Woods Hole, 2003, 8 p, p. 1. 
Para concluir, me parece que hay puntos de encuentro con la profesora Cortina, al menos en la idea de que es absolutamente necesario hoy crear participación y buscar la igualdad, pero no concuerdo con ella en que sea posible a través del mercado capitalista (incluso como medio), desvalorizante, explotador, opresor y enajenante. En tal sentido la salida del conflicto no es ética, o no es meramente ética. No buscamos humanizar el capitalismo a través de un proyecto de la razón cordial (ética cívica dialógica más sentimiento de compasión) ya que "los males del capitalismo sólo pueden ser superados, no rescatando el mercado, sino reemplazándolo" 50 .

Me parece, y en concordancia con lo que ya he dicho, que no podemos usar al mercado, es el mercado el que nos usa, somos sus prisioneros. La construcción de una sociedad nueva, creo, debe apuntar a erradicar la catalaxia ${ }^{51}$, eliminar el juego del mercado en el que nadie es (o más bien quiere hacerse) responsable de la pobreza ni de la desigualdad. Debemos construir un proyecto nuevo, integrativo, democrático y solidario, un proyecto de intercambio pero de no-mercado.

\section{REFERENCIAS BIBLIOGRÁFICAS}

Albert, Michael. Economía participativa. Conferencia en Woods Hole. Ciudad, Estados Unidos: Woods Hole, 2003, 8p.

Callinicos, Alex. An Anti-capitalist Manifesto. Cambridge (MA), Estados Unidos: Polity Press, 2003.

. Igualdad. Madrid, España: Siglo Veintiuno, 2003.

Cortina, Adela. Ciudadanos del mundo: Hacia una teoría de la ciudadanía. Madrid, Espańa: Alianza Editorial, 2001.

Construir confianza. Ética de la empresa en la sociedad de la información y las comunicaciones. Madrid, España: Trotta, 2004.

. Ética de la empresa. Claves para una nueva cultura empresarial. Madrid, Espańa: Trotta, 2000.

La escuela de Francfort. Madrid, España: Cincel, 1992.

Escribar W., Ana. "La ética aplicada, sus condiciones de posibilidad y exigencias a las que responde", en: Revista de Filosofía, No 60, pp. 19-28, Santiago, Chile: Departamento de Filosofía Facultad de Filosofía y Humanidades, Universidad de Chile, 2004.

Friedman, Milton y Friedman, Rose. La libertad de elegir. Hacia un nuevo liberalismo económico. Barcelona, España: Grijalbo, 1980.

Honneth, Axel. "Grounding recognition: a rejoinder to critical questions”, en: Inquiry, Vol. 45, núm. 4 (dic. 2002), pp. 499-512. Londres, Reino Unido: Taylor \& Francis Group, 2002.

. Reconocimiento y menosprecio. Buenos Aires, Argentina: Katz, 2010.

Hopenhayn, Martín. Repensar el trabajo. Historia, profusión y perspectivas de un concepto. Buenos Aires, Argentina: Norma, 2001.

50 Caluinicos, Alex. An Anti-capitalist Manifesto. Cambridge (MA), Estados Unidos: Polity Press, 2003, p. 132.

51 F. A. Hayek le dio este nombre al mercado. 
Lizárraga, Fernando. "Utopías posibles más allá del mercado", en: Otros Logos. Revista de estudios críticos. Año 1, Número 1. Centro de Estudios y Actualización en Pensamiento Político, Decolonialidad e Interculturalidad. Neuquén, Argentina: Universidad Nacional del Comahue, 2010, pp. 70-93.

Lozano, Josep. Ética y empresa. Madrid, Espańa: Trotta, 1999.

Maliandi, Ricardo. Ética: conceptos y problemas. Buenos Aires, Argentina: Biblos, 2004.

MARTínez, Horacio. La ética empresarial al comienzo del nuevo milenio: una aproximación bibliográfica y pedagógica. Bogotá, Colombia: Pontificia Universidad Javeriana, 2005.

Mezquita, Miriam. "Introducción a la Crítica del Agravio Moral", en: Honneth, Axel. Crítica del Agravio Moral. Patologias de la sociedad contemporánea. Buenos Aires, Argentina: Fondo de Cultura Económica, 2009, pp. 9-48.

Ricoeur, Paul. Caminos del reconocimiento. Tres estudios. D. F., México: Fondo de Cultura Económica, 2006.

Robirns, Lionel. Ensayo sobre la Naturaleza y Significación de la Ciencia Económica, En: Ramón, José María (comp.). Recortes para la cátedra de Epistemología. Trelew, Argentina: Universidad Nacional de la Patagonia San Juan Bosco, 2009. 